\title{
The Diagnosis and Treatments of Inguinal Hernia after Radical Prostatectomy
}

\author{
Tao Li, Zhiping Wang* \\ The Institute of Urology, Key Laboratory of Diseases of Urological System, Gansu Province, \\ Gansu Nephro-Urological Clinical Center, Second Hospital of Lanzhou University, Lanzhou, China \\ Email: litao_keai@163.com, ${ }^{*}$ erywzp@lzu.edu.cn
}

Received December 9, 2012; revised January 6, 2013; accepted January 14, 2013

\begin{abstract}
Prostate cancer (PC) is one of the most common causes of cancer-related death in the world among old men. Radical prostatectomy (RP) is the most common surgical procedure in treatments. However, the complications after RRP always confuse surgeons. Urinary incontinence, impotence, erectile dysfunction frequently have effects on the quality of life after RP on patients occurred PC. Inguinal hernias (IHs) after RP is the most common complication, especially indirect hernias. Thus, patients occurred post-IH are frequently performed secondary surgery. In recent years, urologists have explored different surgical techniques, managements, and preoperationly detections to prevent the development of $\mathrm{IH}$ postoperationly. However, the precise mechanism of this procedure occurred is unclear till now. Some retrospective studies have been performed to explore the occurence of IH post-RRP and prophylactic techniques to prevent or decrease IH occurred after RRP. Disappointingly, there is no one efficient and precise method influenced this procedure occurred. We reviewed recent studies about IH after RP through different approaches to evaluate the development of this procedure.
\end{abstract}

Keywords: Inguinal Hernia; Radical Retropubic Prostatectomy; Laparoscopic Radical Prostatectomy

\section{Introduction}

Prostate cancer (PC) is a major worldwide health problem. It is the most commonly diagnosed cancers among old men. The PC incidence is increasing accompanied with increasingly age. Increasing use of prostate-specific antigen (PSA) testing and liberal use of transrectal ultrasound-directed prostate biopsies, prostate cancers are being detected earlier [1,2]. Radical prostatectomy (RP) is today considered gold standard for treating localized PC [3-6]. Radical retropubic prostatectomy (RRP) is primary treatment for clinically organ-confined prostate cancer. The 10-year cancer-specific survival rate for patients with RRP was recently reported to be 96\% [7]. Nonetheless, urinary incontinence, impotence, anastomotic stricture, and erectile dysfunction are frequent complications after RRP, and developing a rectourethral fistula following laparoscopic radical prostatectomy [8-10]. Regan et al. were the first to report that the rate of postprostatectomy inguinal hernias (IH) formation within 6 months was $12 \%$ in 1996 [11]. The incidence thereafter has been reported range from $6.7 \%$ to $38.7 \%$ [12-17], while the overall prevalence rate of $\mathrm{IH}$ in the general male population has been reported to be nearly 5\% [18].

\footnotetext{
"Corresponding author.
}

The precise mechanism of IH after RP is warranted to further investigation. We summarize incidence of $\mathrm{IH}$ after RRP, possible factors, or prophylactic procedure to promote the novel techniques and managements.

\section{Incidence}

Regan et al. [11] initially reported 11 patients of 92 who underwent RRP developed inguinal hernias postoperatively. Ten (91\%) of the hernias were indirect, one (9\%) of the hernias was direct. Akio Matsubara and coworkers recently reported the incidence of postoperative IH after RRP was $10.3 \%$ (32 of 311) with a median follow-up of 36 months. Of the 36 hernias, 28 (78\%), 2 (6\%), and 6 (17\%) were indirect, direct, and unknown, respectively [19]. Inguinal hernias mostly were indirect $[19,20]$. The right-side was dominant in RRP groups [21,22].

Although Regan et al. [11] reported all patients presented with a new complaint of asymptomatic groin bugle within 6 months of their prostatectomy. Kentaro Ichioka et al. [23] reported $81.8 \%$ of postoperative inguinal hernias occurred within 2 years after radical prostatectomy. Fischer and Wanlz [24] recommended that 22\% of 77 inguinal hernias after prostatectomy occurred within the first year and $58 \%$ within the first 2 years.

To prevent post-radical retropubic prostatectomy in- 
guinal hernia, several reports evaluated the incidence of IH through different operation approaches and managements. Shunsuke Yoshimine et al. [22] compared extraperitoneal approach and transperitoneal approach induced postoperative inguinal hernia after laparoscopic radical prostatectomy (LRP). The results showed IH occurred in $4(4.9 \%)$ of the 81 patients in the transperitoneal approach group, and in 37 (9.0\%) of the 412 patients in the extraperitoneal approach group. The overall incidence of inguinal hernia was $8.3 \%$ (41 of 493 patients). Minh Do, M. D. [25] performed 93 patients with endoscopic extraperitoneal radical prostatectomy (EERPE) and observed no hernia recurrence during the follow-up period. Jungle Chi-Hsiang Wu and Johan Stranne et al. [26-28] suggested a lower incidence of postoperative IH after robot-assisted laparoscopic radical prostatectomy (RALP). Akio et al. [19] compared IH after radical perineal prostatectomy (RPP) and RRP, and the incidence was 1.8\% (5 of 285) and 10.3\% (32 of 311) for RPP and RRP, respectively $(\mathrm{P}<0.0001)$. The rate showed by KENTARO ICHIOKA et al. was $21.3 \%, 11.4 \%, 5.4 \%$, respectively. The incidence of IH after radical prostatectomy was rather higher than other two groups [23]. However, on the late report in 2007, the incidence of patients operated with open prostatectomy for benign prostate hyperplasia, pelvic lymph node dissection for staging of prostate cancer, or cystectomy for bladder cancer was no statistically significant [29]. We can conclude that different approaches influenced the incidence of IH after radical prostatectomy and EERPE, RALP, RPP could effectively reduce the IH formation after radical prostatectomy.

\section{Risk Factors}

Although a lot of studies have accessed the risk factors for post-RRP inguinal hernias, a comparison of these is difficult because of factors, the variability in the risk factors evaluated, and varying study methods. However, some investigators postulated it was possible miss some hernias on the preoperative physical examination due to studies were retrospective. In present studies, several clinical factors have been reported to be associated with the inguinal hernia formation postoperatively. Increasing age [30-33], body mass index less than $23 \mathrm{~kg} / \mathrm{m}^{2}$ [23,34], a history of smoking [35], bladder neck contracture (BNC) [30], history of inguinal hernia, [23,30,31,35], and woundrelated problems [35] were identified as significant independent risk factors for the inguinal hernias formation after RP. Previous hernia repair was also a risk factor for the development of IH after RP, with a 3.9-fold increased risk reported by Farhang Rabbani, et al. [36]. Although still others did not identify previous hernia repair as a risk factor [12]. Lodding et al. [30] reported the 2.8-fold increased risk of post-RP inguinal hernia in patients with
BNC was consistent with the increased rate of IH. However, Stranne J. et al. [31] did not consider BNC as a risk factor in their cohort. Farhang Rabbani, et al. [36] supposed the increased intraabdominal pressure from obstruction from the BNC was probably responsible for the increased risk of $\mathrm{IH}$ after RP in patients with BNC. Stranne et al. reported simultaneously pelvic lymph node dissection (PLND), postoperative anastomotic stricture and duration of surgery were not significant risk factors, however, age increases the risk of post-RRP inguinal hernia formation [37]. Some recent researches recommended the surgical time also maybe a major influenced factor $[38,39]$.

The lower mid-line incision has been suggested to be a causative cause [30,40]. CHIA-MING et al. [35] reported the wound-related problems may be one risk factor of post-RRP IH. A postoperative IH incidence of only $2.9 \%$ in a group of 272 patients where RRP was performed through a so called "minilaparotomy" incision of only 6 cm reported by Koie et al. [12]. Matsubara et al. [19] also reported an IH incidence of $1.8 \%$ after RPP, consequently there was no abdominal incision at all, where the whole procedure is performed through a perineal incision. The length of the abdominal incision is of great importance for the postoperative IH formation [12,27]. The development of IH after RRP is likely induced by multifactor preoperatively described above.

\section{Mechanism}

The precise mechanism of IH occured after RP is still unknown. Regan et al. suggested two factors for the development of an inguinal hernia: a patent processus vaginalis and a defect in the internal ring. The internal ring has been postulated to act like a U-shaped valve and that prior to increases in abdominal pressure it acts like a shutter to prevent herniation, the so-called shutter mechanism. Older patients are specifically prone to increased $\mathrm{IH}$, due a small defect in the inguinal ring is unawares enlarged or the nerve supply to the shutter mechanism is injured [11].

The site, myopectineal orifice, is defined superiorly by the internal oblique and transversus abdominus muscles, laterally by the iliopsoas muscle, medially by the rectus muscle, and inferiorly by the pectineal line of the pubis. The orifice is traversed by the spermatic cord and femoral vessels and sealed on its inner surface by the transversalis fascia. Injury of the transversalis fascia may induce failure of the shutter mechanism, which is produced by the transversus aponeurotic arch when the transversus abdominal muscle and internal oblique muscles are stretched. This mechanism strengths the internal ring and plays an important role in maintaining the visceral sac in the myopectineal orifice [23,37]. 
In 2004, Ichioka et al. reported the procedure of exposing of the external iliac vein in PLND during radical prostatectomy often exposes the myopectineal orifice. Therefore, the procedure may injure myopectineal orifice and herniation is more susceptible to development. Furthermore, the vas deferens were cut when the prostate and seminal vesicles were finally exposed when performed radical prostatectomy with a retrograde approach. Thus, the vas deferens probably have been retracted during all radical prostatectomy procedures, also weakening the point of entrance of the vas deferens into the abdominal wall. They postulated prolonged use of the retractor might further damage the fascia, leading to a greater incidence of postoperative IH after radical prostatectomy than that after staging PLND and the postoperative abdominal pressure may affect the incidence of IH [23]. Similar reported by Ichioka et al. [40].

Some studies have suggested the incidence of radical prostatectomy-related inguinal hernia (RPRIH) was lower in cystoprostatectomy group than RRP group [23, 41]. Hypothetically, this may have been associated with the extent of the injury to the transversalis fascia, because this injury will induce failure of the so-called shutter mechanism produced by the transverse aponeurotic arch when the transverse abdominal muscle and internal oblique muscles are stretched, which reinforces the groin $[21,23,42]$. The damage to the transversalis fascia may be less during radical cystoprostatectomy than during RRP [41]. However, Abe et al. regarded stretching injury of the groin region by retraction of the vasa deferentia during prostatectomy as a reasonable explanation, because the incidence of IH after RRP was almost equal to that after LRP [21,37,43]. Several reports have recommended that injury to the abdominal wall structures caused by surgical procedures may trigger subclinical IH that are present before surgery to develop into clinically apparent, bulging ones [35,40,44,45].

In 2007, Akio et al. compared the incidence of IH after RRP and RPP, they discovered the figure of RPP was significantly lower than the former. However, opening of the endopelvic fascia (EPF) appears to be less implicated in IH development, where both the EPF and the dorsal vein complex were left intact in standard RPP, surgical technique was used whereby the EPF was opened followed by division of the dorsal vein complex in modified RPP. However, there was no significantly different in hernia incidence between the standard technique and the modified one [19].

A interesting observation occurred in the laterality of the IH formation. A right-side IH occurred was significantly more often than a left side hernia. Abe et al. [21] reported the right-side dominance of post operative $\mathrm{IH}$ in open RRP group. They suggested that a surgeon who stands on the left side of the patient may result in exces- sive dissection in the right groin area. However, surgeons usually do not retract the vasa deferentia during LRP. Furthermore, Sekita et al. [13] reported that subclinical IH was found in $25 \%$ of RRP patients and the existence of subclinical IH was primarily right-sided (75\%). This could explain the right-side dominance of postoperative IH. However, this is also not a sufficient explanation for the right-side IH dominance. Further investigations were needed to explain why the development of $\mathrm{IH}$ in right side is always more than left side.

\section{Prophylactic Procedures}

The high incidence of $\mathrm{IH}$ is a serious challenge for urologists. Novel prophylactic procedures are urgently needed to prevent the occurrence of IH postoperationly. Johan et al. [27] compared the incidence of IH after RRP and RALP, the results was $12.2 \%, 5.8 \%$, respectively. They suggested that there was significantly difference between the RRP group and the RALP group, and RALP may lower the risks of recurrence. Jungle et al. [26] performed robot-assisted laparoscopic hernioplasty (RALH) during RALP. Therefore, the result was disappointed while there was no significant difference between the RALP group and RALP combined with RALH. Several studies have reported the incidence of IH after RRP is higher than LRP [21,27]. To investigate whether the difference approach of LRP could influence the incidence of IH, Shunsuke et al. [22] compared the development of IH of the extraperitoneal approach (EPA) and transperitoneal approach (TPA) after LRP, they discovered the incidence of IH in the EPA group was significantly greater than TPA group. However, in 2010 Brian M. Lin et al. [46] reported the incidence of IH after LRP was similar, in spite of the laparoscopic approach.

To reduce the incidence of $\mathrm{IH}$ formation, surgeons tried to establish novel and simple methods, managements of preventing post-retropubic prostatectomy inguinal hernia. Yasuyuki S. et al. [47] dissected the peritoneum at the internal inguinal ring and isolated the spermatic cord from surrounding the peritoneum, beginning at the internal inguinal ring and proceeding towards the iliac fossa, using the preperitoneal approach. They observed the incidence of $\mathrm{IH}$ in the prophylactic procedure group (1.6\%) was significantly lower than that in the conventinoal RRP group (50\%) during a median follow-up period of 41 months indicating the efficacy of the procedure. Yasuhisa F. et al. [48] isolated the spermatic cords and dissected the vas deferens, leading to destruction of the spermatic sheath covering the spermatic cord. The results showed that this measure was safe and effective to prevent IH formation after RRP. Kazumi Taguchi et al. [43] released the bilateral spermatic cord from the peritoneum without placing mesh sheets and manually 
without ligating the vaginal process of the peritoneum at least $5 \mathrm{~cm}$ from the abdominal wall. Thus, there was no IH occurred in the procedure group. In conclusion, isolating the spermatic cord may be a sufficient method to prevent IH post-RRP. However, longer-term observation of more patients are warranted to reveal whether the prophylactic method could efficiently prevent the development of IH after RRP.

In 2004, TEBER et al. [49] indicated performing LRP plus repair of IH using prosthetic mesh or LRP was no significantly difference in the incidence of IH. Either the transperitoneal or extraperitoneal approach is feasible. In recent studies, many investigators showed the similar conclusion [25,37,50]. Although, the laparoscopic totally extraperitoneal after radical prostatectomy could be influenced by surgeons, skill and experience recommended by J.-L. et al. [51].

Lepor et al. [52] detected an inguinal hernia by physical examination and routine abdominal computed tomography preoperatively in 1130 patients, consequently, 146 (13\%) had a preoperative inguinal hernia. The sensitivity was $96.3 \%$ and $42.5 \%$, respectively. All candidates for open RRP should undergo a careful physical examination to identify asymptomatic inguinal hernias. Their findings demonstrated that the previously reported high incidence of symptomatic inguinal hernias that developed after open RRP can be explained by a failure to diagnose preexisting inguinal hernias and the development of new hernias. Fukuta et al. [41] identified subclinical IH in 20 (20.4\%) of 98 patients who had undergone RRP on the preoperative CT images, with a great incidence (60.6\%) IH within 12 months. The surgeons should attach importance to subclinical IH examined by CT scans.

\section{Conclusion}

The inguinal hernia is a common complication after RP. However, the risk factors and mechanism are not precisely explained till now. There is still no one efficient method to prevent the development of IH after RP. Some randomized clinical trials should be taken to explore this procedure. Further study of preventing the IH occurred is urgently needed.

\section{REFERENCES}

[1] G. L. Lu-Yao, D. McLerran, J. Wasson and J. E. Wennberg, “An Assessment of Radical Prostatectomy. Time Trends, Geographic Variation, and Outcomes. The Prostatic Outcomes Research Team," Journal of the American Medical Association, Vol. 269, 1993, pp. 26762677. doi:10.1001/jama.1993.03500200047031

[2] A. V. Sarma and D. Schottenfeld, "Prostate Cancer Incidence, Mortality, and Survival Trends in the United States: 1981-2001,” Seminars in Urologic Oncology, Vol.
20, No. 1, 2002, pp. 3-9. doi:10.1053/suro.2002.30390

[3] J. Hugosson, J. Stranne and S. V. Carlsson, "Radical Retropubic Prostatectomy: A Review of Outcomes and SideEffects,” Acta Oncologica, Vol. 50, No. S1, 2011, pp. 9297. doi:10.3109/0284186X.2010.535848

[4] E. Xylinas, A. Daché and M. Rouprêt, "Is Radical Prostatectomy a Viable Therapeutic Option in Clinically Locally Advanced (cT3) Prostate Cancer?” BJU International, Vol. 106, No. 11, 2010, pp. 1596-1600. doi:10.1111/j.1464-410X.2010.09630.x

[5] P. C. Walsh, “Anatomic Radical Prostatectomy: Evolution of the Surgical Technique,” Journal of Urology, Vol. 160, No. 6, 1998, pp. 2418-2424. doi:10.1016/S0022-5347(01)62202-X

[6] M. Wirth, "Radical Prostatectomy-The Gold Standard in the Treatment of Localised and Locally Advanced Prostate Cancer,” European Journal of Cancer, Vol. 47, Suppl. 1, 2011, p. S49.

[7] M. Han, A. W. Partin, C. R. Pound, et al., "Long-Term Biochemical Disease-Free and Cancer-Specific Survival Following Anatomic Radical Retropubic Prostatectomy: The 15-Year Johns Hopkins Experience,” Urologic Clinics of North America, Vol. 28, No. 3, 2001, pp. 555-565. doi:10.1016/S0094-0143(05)70163-4

[8] L. Chun and M. A. Abbas, "Rectourethral Fistula Following Laparoscopic Radical Prostatectomy,” Techniques in Coloproctology, Vol. 15, No. 3, 2011, pp. 297-300. doi:10.1007/s10151-011-0710-8

[9] J. Maarouf, J. Ghorbel, Z. Gammoudi, et al., "Anastomotic Stricture after Radical Prostatectomy (about 7 Cases)," Urology, Vol. 76, No. 3, 2010, p. S103.

[10] L. Glickman, G. Godoy and H. Lepor, "Changes in Continence and Erectile Function between 2 and 4 Years after Radical Prostatectomy,” Journal of Urology, Vol. 181, No. 2, 2009, pp. 731-735. doi:10.1016/j.juro.2008.10.019

[11] T. C. Regan, R. M. Mordkin, N. L. Constantinople, et al., "Incidence of Inguinal Hernias Following Radical Retropubic Prostatectomy,” Urology, Vol. 47, No. 4, 1996, pp. 536-537. doi:10.1016/S0090-4295(99)80491-9

[12] T. Koie, T. Yoneyama, N. Kamimura, et al., "Frequency of Postoperative Inguinal Hernia after Endoscope-Assisted Mini-Laparotomy and Conventional Retropubic Radical Prostatectomies,” International Journal of Urology, Vol. 15, No. 3, 2008, pp. 226-229. doi:10.1111/j.1442-2042.2007.01983.x

[13] N. Sekita, H. Suzuki, S. Kamijima, K. Chin, M. Fujimura, K. Mikami, et al., "Incidence of Inguinal Hernia after Prostate Surgery: Open Radical Retropubic Prostatectomy versus Open Simple Prostatectomy versus Transurethral Resection of the Prostate," International Journal of Urology, Vol. 16, No. 1, 2009, pp. 110-113. doi:10.1111/j.1442-2042.2008.02190.X

[14] P. J. Tsai, C. C. Yu, Y. H. Lee and J. K. Huang, "Inguinal Hernia after Radical Retropubic Prostatectomy-Experience of Kaohsiung Veterans General Hospital,” Journal of the Chinese Medical Association, Vol. 67, No. 3, 2004, pp. 141-144.

[15] T. Nomura, H. Mimata, H. Kitamura, et al., "Lower Inci- 
dence of Inguinal Hernia: Minilaparotomy Radical Retropubic Prostatectomy Compared with Conventional Technique. A Preliminary Report,” Urologia Internationalis, Vol. 74, No. 1, 2005, pp. 32-37. doi:10.1159/000082706

[16] J. Stranne, J. Hugosson, P. Iversen, et al., "Inguinal Hernia in Stage M0 Prostate Cancer: A Comparison of Incidence in Men Treated with and without Radical Retropubic Prostatectomy-An Analysis of 1105 Patients," Urology, Vol. 65, No. 5, 2005, pp. 847-851.

doi:10.1016/j.urology.2004.11.014

[17] M. Sun, G. Lughezzani, A. Alasker, et al., "Comparative Study of Inguinal Hernia Repair after Radical Prostatectomy, Prostate Biopsy, Transurethral Resection of the Prostate or Pelvic Lymph Node Dissection,” Journal of Urology, Vol. 183, No. 3, 2010, pp. 970-975. doi:10.1016/j.juro.2009.11.036

[18] I. M. Rutkow, "Epidemiologic, Economic, and Sociologic Aspects of Hernia Surgery in the United States in the 1990s,” Surgical Clinics of North America, Vol. 78, No. 6, 1998, pp. 941-951. doi:10.1016/S0039-6109(05)70363-7

[19] A. Matsubara, T. Yoneda, T. Nakamoto, et al., "Inguinal Hernia after Radical Perineal Prostatectomy: Comparison with the Retropubic Approach,” Urology, Vol. 70, No. 6, 2007, pp. 1152-1156.

[20] J.-L. Dulucq, P. Wintringer and A. Mahajna, "Occult Hernias Detected by Laparoscopic Totally Extra-Peritoneal Inguinal Hernia Repair: A Prospective Study,” Hernia, Vol. 15, No. 4, 2011, pp. 399-402. doi:10.1007/s10029-011-0795-z

[21] A. Takashige, N. Shinohara, T. Harabayashi, et al., "Postoperative Inguinal Hernia after Radical Prostatectomy for Prostate Cancer,” Urology, Vol. 69, No. 2, 2007, pp. 326- 329.

[22] S. Yoshimine, A. Miyajima, K. Nakagawa, et al., "Extraperitoneal Approach Induces Postoperative Inguinal Hernia Compared with Transperitoneal Approach after Laparoscopic Radical Prostatectomy,” Japanese Journal of Clinical Oncology, Vol. 40, No. 4, 2010, pp. 349-352. doi:10.1093/jjco/hyp172

[23] K. Ichioka, K. Yoshimura, N. Utsunomiya, et al., "High Incidence of Inguinal Hernia after Radical Retropubic Prostatectomy," Urology, Vol. 63, No. 2, 2003, pp. 278281.

[24] E. Fischer and G. E. Wantz, "Radical Retropubic Prostatectomy and Groin Hernia: Cause and Effect?” Hernia, Vol. 1, No. 2, 1997, pp. 67-70. doi:10.1007/BF02427663

[25] M. Do, E. N. Liatsikos, P. Kallidonis, et al., "Hernia Repair during Endoscopic Extraperitoneal Radical Prostatectomy: Outcome after 93 Cases,” Journal of Endourology, Vol. 25, No. 4, 2011, pp. 625-629.

[26] J. C.-H. Wu, Y.-C. Ou, H.-S. Wu, et al., "Inguinal Hernias in Robotic-Assisted Laparoscopic Radical Prostatectomy: A Surgeon's Experience," Formosan Journal of Surgery, Vol. 44, No. 4, 2011, pp. 141-145. doi:10.1016/j.fjs.2011.08.008

[27] J. Stranne, E. Johansson, A. Nilsson, et al., "Inguinal Hernia after Radical Prostatectomy for Prostate Cancer: Results from a Randomized Setting and a Nonrandomized Setting," European Urology, Vol. 58, No. 5, 2010, pp.
719-726. doi:10.1016/j.eururo.2010.08.006

[28] A. Mottrie, G. De Naeyer, G. Novara and V. Ficarra, "Robotic Radical Prostatectomy: A Critical Analysis of the Impact on Cancer Control," Current Opinion in Urology, Vol. 21, No. 3, 2011, pp. 179-184. doi:10.1097/MOU.0b013e328344e53e

[29] J. Stranne, J. Hugosson and P. Lodding, “Inguinal Hernia Is a Common Complication in Lower Midline Incision Surgery,” Heinia, Vol. 11, No. 3, 2007, pp. 247-252.

[30] P. Lodding, C. Bergdahl, M. Nyberg, et al., "Inguinal Hernia after Radical Retropubic Prostatectomy for Prostate Cancer: A Study of Incidence and Risk Factors in Comparison to No Operation and Lymphadenectomy," Journal of Urology, Vol. 166, No. 3, 2001, pp. 964-967. doi:10.1016/S0022-5347(05)65874-0

[31] J. Stranne, J. Hugosson and P. Lodding, "Post-Radical Retropubic Prostatectomy Inguinal Hernia: An Analysis of Risk Factors with Special Reference to Preoperative Inguinal Hernia Morbidity and Pelvic Lymph Node Dissection," Journal of Urology, Vol. 176, No. 5, 2006, pp. 2072-2076. doi:10.1016/j.juro.2006.07.007

[32] S. M. H. Alibhai, M. Leach, G. Tomlinson, et al., “30Day Mortality and Major Complications after Radical Prostatectomy: Influence of Age and Comorbidity,” Journal of the National Cancer Institute, Vol. 97, No. 20, 2005, pp. 1525-1532. doi:10.1093/jnci/dji313

[33] B. Lppenberg, J. Noldus, A. Holz, et al., "Reporting Complications after Open Radical Retropubic Prostatectomy Using the Martin Criteria,” Journal of Urology, Vol. 184, No. 3, 2010, pp. 944-948. doi:10.1016/j.juro.2010.05.032

[34] F. Giuseppe, R. Oreste, R. Michele, et al., "Correlation between Detrusor Hypocontractility and Bladder Neck Obstruction after Radical Prostatectomy,” Neurourology and Urodynamics, Vol. 29, Suppl. 2, 2010, p. 58.

[35] C. M. Twu, Y. C. Ou, C. R. Yang, et al., "Predicting Risk Factors for Inguinal Hernia after Radical Retropubic Prostatectomy," Urology, Vol. 66, No. 4, 2005, pp. 814-818. doi:10.1016/j.urology.2005.04.034

[36] F. Rabbani, L. H. Yunis, K. Touijer, et al., "Predictors of Inguinal Hernia after Radical Prostatectomy,” Urology, Vol. 77, No. 2, 2011, pp. 391-395. doi:10.1016/j.urology.2010.04.019

[37] M. E. Nielsen and P. C. Walsh, "Systematic Detection and Repair of Subclinical Inguinal Hernias at Radical Reteropubic Prostatectomy,” Urology, Vol. 66, No. 5, 2005, pp. 1034-1037.

[38] H. Lepr and L. Kaci, "Contemporary Evaluation of Operative Parameters and Complications Related to Open Radical Retropubic Prostatectomy,” Urology, Vol. 62, No. 4, 2003, pp. 702-706. doi:10.1016/S0090-4295(03)00515-6

[39] V. Klevecka, L. Burmester, M. Musch, et al., "Intraoperative and Early Postoperative Complications of Radical retropubic Prostatectomy," Urologia Internationalis, Vol. 79, No. 3, 2007, pp. 217-225. doi:10.1159/000107953

[40] K. Ichioka, N. Kohei, K. Yoshimura, Y. Arai and A. Terai, "Impact of Retraction of Vas Deferens in Postradical Prostatectomy Inguinal Hernia,” Urology, Vol. 70, No. 
3, 2007, pp. 511-514. doi:10.1016/j.urology.2007.04.025

[41] F. Fukuta, S.-I. Hisasue, M. Yanase, et al., "Preoperative Computed Tomography Finding Predicts for Postoperative Inguinal Hernia: New Perspective for Radical Prostatectomy-Related Inguinal Hernia,” Urology, Vol. 68, No. 2, 2006, pp. 267-271.

[42] G. E. Wantz, "Giant Prosthetic Reinforcement of the Visceral Sac: The Stoppa Groin Hernia Repair,” Surgical Clinics of North America, Vol. 78, No. 6, 1998, pp. 10751087. doi:10.1016/S0039-6109(05)70370-4

[43] K. Taguchi, T. Yasui, H. Kubota, et al., "Simple Method of Preventing Postoperative Inguinal Hernia after Radical Retropubic Prostatectomy,” Urology, Vol. 76, No. 5, 2010, pp. 1083-1087. doi:10.1016/j.urology.2010.05.002

[44] G. Lughezzani, M. Sun, P. Perrotte, et al., "Comparative Study of Inguinal Hernia Repair Rates after Radical Prostatectomy or External Beam Radiotherapy," International Journal of Radiation Oncology, Biology, Physics, Vol. 78, No. 5, 2010, pp. 1307-1313. doi:10.1016/j.ijrobp.2009.09.065

[45] M. E. Nielsen and P. C. Walsh, "Systematic Detection and Repair of Subclinical Inguinal Hernias at Radical Retropubic Prostatectomy,” Urology, Vol. 66, No. 5, 2005, pp. 1034-1037. doi:10.1016/j.urology.2005.05.028

[46] B. M. Lin, M. E. Hyndman, et al., "Incidence and Risk Factors for Inguinal and Incisional Hernia after Laparoscopic Radical Prostatectomy,” Urology, Vol. 77, No. 4, 2011, pp. 957-962. doi:10.1016/j.urology.2010.12.011
[47] Y. Sakai, T. Okuno, T. Kijima, et al., "Simple Prophylactic Procedure of Inguinal Hernia after Radical Retropubic Prostatectomy: Isolation of the Spermatic Cord," International Journal of Urology, Vol. 16, No. 10, 2009, pp. 848-851. doi:10.1111/j.1442-2042.2009.02370.x

[48] Y. Fujii, S. Yamamoto, J. Yonese, et al., "A Novel Technique to Prevent Postradical Retropubic Prostatectomy Inguinal Hernia: The Processus Vaginalis Transection Method,” Urology, Vol. 75, No. 3, 2010, pp. 713-717. doi:10.1016/j.urology.2009.05.051

[49] D. Teber, T. Erdogru, D. Zukosky, et al., "Prosthetic Mesh Hernioplasty during Laparoscopic Radical Prostatectomy,” Urology, Vol. 65, No. 6, 2005, pp. 1173-1178. doi:10.1016/j.urology.2004.12.063

[50] D. T. Saint-Elie and F. F. Marshall, "Impact of Laparoscopic Inguinal Hernia Repair Mesh on Open Radical Retropubic Prostatectomy,” Urology, Vol. 76, No. 5, 2010, pp. 1078-1082. doi:10.1016/j.urology.2010.01.015

[51] J.-L. Dulucq, P. Wintringer and A. Mahajna, "Totally Extraperitoneal (TEP) Hernia Repair after Radical Prostatectomy or Previous Lower Abdominal Surgery," Surgical Endoscopy and Other Interventional Techniques, Vol. 20, No. 3, 2006, pp. 473-476. doi:10.1007/s00464-006-3027-3

[52] H. Lepor and D. Bobbins, "Inguinal Hernias in Men Undergoing Open Radical Retropubic Prostatectomy,” Urology, Vol. 70, No. 5, 2007, pp. 961-964. 